\title{
Sarcopenia and sarcopenic obesity
}

\author{
Kyung Mook Choi
}

Division of Endocrinology and Metabolism, Department of Internal Medicine, Korea University College of Medicine, Seoul, Korea

Received: June 22, 2016 Accepted: October 23, 2016

\section{Correspondence to} Kyung Mook Choi, M.D. Division of Endocrinology and Metabolism, Department of Internal Medicine, Korea University College of Medicine, 73 Inchon-ro, Seongbuk-gu, Seoul 02841, Korea Tel: $+82-2-2626-3043$

Fax: +82-2-2626-1096

E-mail:medica7@gmail.com
Sarcopenia is an age-associated loss of muscle mass and decline in muscle strength; it is common in older adults and is associated with significant morbidity and mortality. Despite its prevalence, there is currently no universally adopted definition of sarcopenia. In addition to low muscle mass measurements, recent research has recognized the importance of muscle strength and physical performance. Aging induces changes in body composition, such as an increase in visceral fat and reduced muscle mass. Recently, the new concept of sarcopenic obesity has emerged, reflecting a combination of sarcopenia and obesity. The rapidly increasing prevalence and serious consequences of sarcopenic obesity are recognized as a critical public health risk in the aging society. Sarcopenia and obesity share several pathophysiological mechanisms, and they may potentiate each other. The present paper reviews the definitions and techniques used to measure sarcopenia, as well as the health outcomes of sarcopenic obesity. It also highlights the role of diminished muscle mass and strength in cardiometabolic disease mortality. Additional research may be needed to promote the identification and management of sarcopenia and sarcopenic obesity in the elderly population.

Keywords: Sarcopenia; Obesity; Aging; Muscles

\section{INTRODUCTION}

One of the dramatic changes associated with human aging is the progressive decline of skeletal muscle mass. Several prospective studies have suggested that muscle mass decreases by approximately $6 \%$ per decade after mid-life [1]. Interestingly, results from the Health Aging, and Body Composition (ABC) study showed that the decreased muscle strength is predominantly due to a lower muscle mass [2]. There are significant differences among individuals in peak muscle mass, the age at which muscle loss begins, and the amount of muscle that is lost over time [3]. Rosenberg first proposed the term "sarcopenia," which originated from the Greek words sarx (flesh) and penia (loss), to refer to the age-related loss of skeletal muscle mass [4]. At the cellular level, sarcopenia is accompanied by a loss of innervation and adaptive changes in the proportions of slow and fast motor units, as well as in the cross-sectional area of muscle fibers [5]. Primary sarcopenia is the term used to define sarcopenia that is caused by aging itself, whereas secondary sarcopenia describes sarcopenia that is caused by disuse (immobility or physical inactivity), disease (advanced organ failure, malignancy, neurodegenerative, or endocrine diseases), and inadequate nutrition [6]. Sarcopenia is closely associated with frailty, physical disability, hospitalization, osteoporosis, osteoarthritis, and even mortality $[7,8]$.

\section{DEFINITIONS OF SARCOPENIA}

Several different definitions of sarcopenia have been suggested, although no consensus definition has been adopted [9]. In 1998, Baumgartner et al. [10] first defined sarcopenia as an appendicular skeletal muscle mass 
(ASM) $(\mathrm{kg}) /$ height $^{2}\left(\mathrm{~m}^{2}\right)$ of less than two standard deviations (SDs) below the mean of a young reference group. Janssen et al. [11] adopted the skeletal muscle mass index $(\mathrm{SMI}=$ skeletal muscle mass/body mass $\times 100)$ to establish the prevalence of sarcopenia in older Americans. They considered subjects with an SMI within one to two SDs of young adult values to have class I sarcopenia, and those with an SMI below two SDs of young adult values to have class II sarcopenia. In the Health $A B C$ study, Newman et al. [12] proposed an alternative definition of sarcopenia using appendicular lean mass (ALM) adjusted for height and body fat mass (residuals). They indicated that Baumgartner's definition (ASM/height) ${ }^{2}$ is strongly correlated with body mass index (BMI), and therefore identifies fewer obese individuals as sarcopenic. To examine the prevalence of sarcopenia in Korean adults and explore its impact on health outcomes, we previously established a cohort study called the Korean Sarcopenic Obesity Study (KSOS) [13]. The prevalence of sarcopenia differed according to the definition applied, as well as age and sex.

Since then, the definition of sarcopenia has evolved to highlight muscle strength and physical performance. The European Working Group on Sarcopenia in Older People (EWGSOP) defined sarcopenia as the presence of both low muscle mass and low muscle strength or performance [6]. Recently, the Foundation for the National Institutes of Health (FNIH) sarcopenia project suggested using ALM with adjustment for BMI to define low muscle mass [14]. Using a dataset from nine large observational studies containing more than 25,000 participants, the FNIH sarcopenia project established ALM/ BMI ratio cutoff values of $<0.789$ for men and $<0.512$ for women [14,15]. Recently, Kim et al. [16] compared skeletal muscle mass indices and described their clinical implications. Further studies might be needed to compare the definitions of sarcopenia with regard to their impact on disability, cardiometabolic risk profiles, and mortality.

\section{TECHNIQUES FOR ASSESSING SARCOPENIA}

Various methods can be used to assess muscle mass and strength (Table 1). Anthropometric measurements, such as mid-upper arm circumference, calf circumference, and skin fold thickness, are not recommended for diagnosing sarcopenia since they are prone to error [6]. Computed tomography (CT) and magnetic resonance imaging are able to effectively distinguish fat from other soft tissues, which makes these the standard techniques for evaluating muscle mass in research [6]. However, limited access, the high cost, and the risk of radiation (with CT) preclude the wider use of these techniques in clinical practice. Therefore, dual energy X-ray absorptiometry (DXA) is the preferred method for correctly evaluating body composition; it is widely used to assess muscle mass in research studies because of good precision and safety with relatively low radiation [7]. Therefore, measuring ALM using DXA has become a standard criterion in most current sarcopenia definitions. Bioimpedance analysis (BIA) is an inexpensive, easy to use, and reproducible method that is considered a portable alternative to DXA. However, neither DXA nor BIA can distinguish between extracellular and intracellular water, making these techniques prone to error depending on the hydration status of the patient [7].

Muscle strength is commonly evaluated using handgrip strength, which is an easy, reliable, and inexpensive method for identifying elderly adults at risk for disability [17]. Based on the EWGSOP definition, cutoffs for grip strength are $<20 \mathrm{~kg}$ for women and $<30 \mathrm{~kg}$ for men [6]. In contrast, the FNIH sarcopenia project established cutoffs of $<16 \mathrm{~kg}$ for women and $<26 \mathrm{~kg}$ for men [14,15]. Knee flexion techniques are appropriate for research

Table 1. Methods for measurement of muscle mass, muscle strength, and physical performance

\begin{tabular}{lll}
\hline Muscle mass & \multicolumn{1}{c}{ Muscle strength } & Physical performance \\
\hline Anthropometry & Handgrip strength & Short physical performance battery \\
Computed tomography & Knee flexion/extension & Usual gait speed \\
Magnetic resonance imaging & & Timed get-up-and-go test \\
$\begin{array}{l}\text { Dual energy X-ray absorptiometry } \\
\text { Bioimpedance analysis }\end{array}$ & \\
\hline
\end{tabular}


purposes; however, their use in clinical practice is limited by the need for specific machines. The short physical performance battery (SPPB) is used in both clinical and research settings to measure physical performance. It combines gait speed, chair-rise time, and balance assessment to generate a standard measurement [18]. Usual gait speed, which is part of the SPPB, might be adopted as a single parameter to provide a predictive value for disability [19]. Previous studies have demonstrated that a walking speed of less than either 1.0 or $0.8 \mathrm{~m} / \mathrm{sec}$ is associated with adverse outcomes [20]. Timed get-upand-go examines the time needed to accomplish a series of functionally critical tasks; it can also serve as a performance measurement.

\section{MECHANISMS OF SARCOPENIA}

Several underlying mechanisms have been linked to the development of sarcopenia, although not all have been fully elucidated. The relative contribution of the different mechanisms may vary over time in an individual with sarcopenia [6]. Understanding the mechanisms underlying sarcopenia may provide strategies for intervention and disease improvement. Most mechanisms of sarcopenia are also associated with visceral obesity, which may lead to a vicious cycle of intricate interactions among risk factors. Insulin resistance plays an important role in muscle fiber atrophy and mitochondrial dysfunction [21]. Bijlsma et al. [22] demonstrated that insulin resistance is related distinctly to the different diagnostic criteria for sarcopenia; it is better reflected by relative muscle mass than by absolute muscle mass or muscle strength. Aging is related to changes in a variety of hormones, including testosterone, estrogen, growth hormone, insulin-like growth factor 1 , and corticosteroids [23]. These hormonal changes may affect the anabolic and catabolic processes in skeletal muscle [24]. Reduced androgen and estrogen concentrations decrease muscle mass and strength. In addition, previous studies have suggested that sarcopenia is an inflammatory state that is driven by proinflammatory cytokines and oxidative stress [25]. Oxidative stress modulates the expression of transcription factors, such as nuclear factor- $\mathrm{\kappa B}$, which enhances proteolytic pathways and increases the production of proinflammatory cytokines [26]. Tumor necrosis factor $\alpha$ impairs protein synthesis in skeletal muscle by altering translation initiation, which may contribute to sarcopenia [27]. In a prospective, population-based study, higher levels of interleukin 6 and C-reactive protein were associated with a greater decline in muscle strength [28]. Another pivotal factor in the regulation of skeletal muscle mass is myostatin, also known as growth/differentiation factor 8 , which inhibits muscle cell growth and differentiation [29]. Deletion of the myostatin gene causes a double-muscled phenotype in cattle [30]. Myostatin has emerged as a potential mediator of sarcopenia and a promising therapeutic target [31].

\section{IMPLICATIONS OF THE EFFECTS OF SARCOPE- NIA ON CARDIOMETABOLIC RISK AND MOR- TALITY}

Sarcopenia is independently associated with insulin resistance [32], and in the KSOS study, type 2 diabetes was independently associated with an increased risk of sarcopenia [33]. Diabetes has been suggested to be an intermediate step in the development of frailty in individuals with sarcopenia [34]. Furthermore, we previously found that individuals with a lower muscle mass have an increased risk of nonalcoholic fatty liver disease, which is now recognized as a feature of metabolic syndrome [35]. Low muscle mass is related to cardiovascular risk factors including hypertension and arterial stiffness $[36,37]$. The coexistence of sarcopenia and metabolic syndrome further aggravates the risk of cardiovascular risk factors such as type 2 diabetes, hypertension, and hyperlipidemia in adult Japanese women [38]. Previous prospective studies have consistently shown a relationship between low muscle mass or muscle strength and an increased risk of mortality [39-41]. In the British Regional Heart Study, Atkins et al. [42] reported that sarcopenia is associated with greater cardiovascular mortality and allcause mortality. Hirani et al. [43] showed that sarcopenia, as defined by the FNIH criteria, is associated with an increased risk of mortality, disability, and institutionalization in community-dwelling older men. Several studies have suggested that deteriorated muscle strength is a more important risk factor for mortality than a decline in muscle mass $[44,45]$. 


\section{SARCOPENIC OBESITY}

Obesity is an important health threat that is a major risk factor for metabolic and cardiovascular morbidity and mortality. The prevalence of obesity in middle-aged and older adults has doubled since 1980, and it continues to increase worldwide [9]. The major age-related changes in body composition include an increase in body fat and a decline in skeletal muscle, although BMI may remain relatively unchanged. Sarcopenic obesity was first defined by Baumgartner [46] as the co-presence of sarcopenia and obesity, as measured using DXA. We subsequently introduced the ratio of visceral fat to thigh muscle area (VMR), as measured using CT, as a single indicator of sarcopenic obesity [47]. We found that VMR values were independently associated with metabolic syndrome in Korean adults.

The complex interplay of common pathophysiological mechanisms, such as increased proinflammatory cytokines, oxidative stress, insulin resistance, and hormonal changes and decreased physical activity, underlie the close relationship between sarcopenia and obesity (Fig. 1). We reported that the homoeostasis model assessment of insulin resistance (HOMA-IR) and vitamin $\mathrm{D}$ levels are independently associated with sarcopenic obesity in men, whereas HOMA-IR and high-sensitivity C-reactive protein are associated with sarcopenic obesity in women [48]. A vicious cycle may exist between the accumulation of ectopic fat and the loss of skeletal muscle mass since they have a reciprocal influence on each

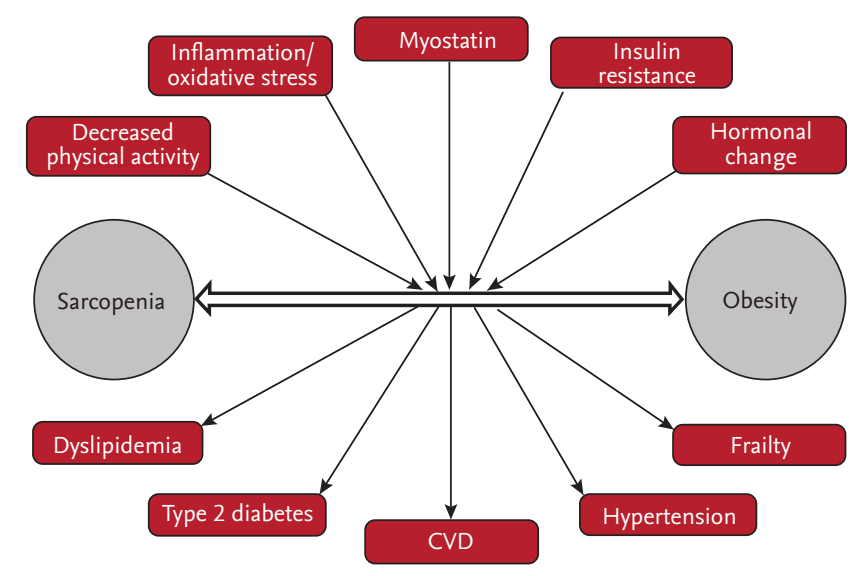

Figure 1. Mechanisms and consequences of sarcopenia and obesity. CVD, cardiovascular disease. other [49]. Sarcopenia reduces physical activity, which leads to decreased energy expenditure and increases the risk of obesity [50]. In contrast, an increase in visceral fat induces inflammation, which contributes to the development of sarcopenia [51]. In our longitudinal study, visceral obesity was independently associated with the future loss of skeletal muscle mass after adjusting for confounding factors [52].

\section{IMPACT OF SARCOPENIC OBESITY ON MORBIDITY AND MORTALITY}

Both sarcopenia and obesity are associated with metabolic disorders, morbidity, and mortality [50]. Thus, it has been hypothesized that sarcopenic obesity may have a greater impact on metabolic diseases and cardiovascular morbidity and mortality than either sarcopenia or obesity alone [9,53].

Recent studies have emphasized the influence of sarcopenic obesity on cardiometabolic risk and health outcomes [23,53,54]. Several cross-sectional studies in elderly Koreans have demonstrated that individuals with sarcopenic obesity have worse cardiovascular risk profiles, including hyperglycemia, hypertension, dyslipidemia, insulin resistance, and lower cardiorespiratory fitness [55-57]. Similarly, a Taiwanese study showed that sarcopenic obesity is associated with the highest risk of metabolic syndrome [58]. Furthermore, sarcopenia exacerbated obesity-associated dysglycemia and insulin resistance in a cross-sectional study from the National Health and Nutrition Examination Survey III (NHANES III) [32]. However, there have been conflicting results regarding whether patients with sarcopenic obesity have the worst risk profiles. Several cross-sectional studies have reported that obese individuals have more cardiovascular risk factors than those with sarcopenic obesity $[59,60]$.

A limited number of studies have investigated the effects of sarcopenia and obesity on cardiovascular disease (CVD) and mortality. Stephen and Janssen [61] showed that sarcopenic obesity is associated with increased CVD risk based on muscle strength but not muscle mass. In the British Regional Heart Study, subjects with sarcopenic obesity had a significantly higher risk of mortality compared to nonsarcopenic, nonobese subjects [41]. 
In contrast, in a study using data from the NHANES III study, older women with sarcopenia had a higher risk of all-cause mortality, independent of obesity [62]. A recent meta-analysis demonstrated that sarcopenic obesity is associated with a $24 \%$ increase in the risk of all-cause mortality compared to patients without sarcopenic obesity, particularly in men [63].

\section{CONCLUSIONS}

Although BMI is a simple estimator of obesity, it cannot fully reflect muscle mass and body fat. Accumulating evidence underscores the need to consider muscle function and mass when evaluating the risk of obesity in elderly people. Visceral fat and muscle mass seem to have opposing influences on cardiometabolic morbidity and mortality. Sarcopenic obesity is a relatively novel concept that has become increasingly important in the aging population. There has been some evidence that sarcopenic obesity may be associated with an increased risk of mortality and cardiovascular risk factors compared to sarcopenia or obesity alone. However, several different definitions of sarcopenia limit the clinical application of sarcopenia and sarcopenic obesity with regard to metabolic disorders and CVD. A consensus definition of sarcopenia is needed to promote the standardized diagnosis and management of sarcopenic obesity. Furthermore, in addition to reducing body fat, increasing muscle mass and strength is required to promote healthy aging.

\section{Conflict of interest}

No potential conflict of interest relevant to this article was reported.

\section{Acknowledgments}

The present work was supported by the Basic Science Research Program through the National Research Foundation of Korea (NRF), which is funded by the Ministry of Education, Science and Technology (2015R1D1A1A09057389).

\section{REFERENCES}

1. Janssen I. Evolution of sarcopenia research. Appl Physiol Nutr Metab 2010;35:707-712.
2. Newman AB, Haggerty CL, Goodpaster B, et al. Strength and muscle quality in a well-functioning cohort of older adults: the Health, Aging and Body Composition Study. J Am Geriatr Soc 2003;51:323-330.

3. Janssen I. The epidemiology of sarcopenia. Clin Geriatr Med 2011;27:355-363.

4. Rosenberg IH. Sarcopenia: origins and clinical relevance. J Nutr 1997;127(5 Suppl):990S-991S.

5. Lang T, Streeper T, Cawthon P, Baldwin K, Taaffe DR, Harris TB. Sarcopenia: etiology, clinical consequences, intervention, and assessment. Osteoporos Int 2010;21:543559.

6. Cruz-Jentoft AJ, Baeyens JP, Bauer JM, et al. Sarcopenia: European consensus on definition and diagnosis: Report of the European Working Group on Sarcopenia in Older People. Age Ageing 2010;39:412-423.

7. Edwards $\mathrm{MH}$, Buehring B. Novel approaches to the diagnosis of sarcopenia. J Clin Densitom 2015;18:472-477.

8. Kim HT, Kim HJ, Ahn HY, Hong YH. An analysis of age-related loss of skeletal muscle mass and its significance on osteoarthritis in a Korean population. Korean J Intern Med 2016;31:585-593.

9. Wannamethee SG, Atkins JL. Muscle loss and obesity: the health implications of sarcopenia and sarcopenic obesity. Proc Nutr Soc 2015;74:405-412.

10. Baumgartner RN, Koehler KM, Gallagher D, et al. Epidemiology of sarcopenia among the elderly in New Mexico. Am J Epidemiol 1998;147:755-763.

11. Janssen I, Heymsfield SB, Ross R. Low relative skeletal muscle mass (sarcopenia) in older persons is associated with functional impairment and physical disability. J Am Geriatr Soc 2002;50:889-896.

12. Newman AB, Kupelian V, Visser M, et al. Sarcopenia: alternative definitions and associations with lower extremity function. J Am Geriatr Soc 2003;51:1602-1609.

13. Kim TN, Yang SJ, Yoo HJ, et al. Prevalence of sarcopenia and sarcopenic obesity in Korean adults: the Korean Sarcopenic Obesity Study. Int J Obes (Lond) 2009;33:885-892.

14. Studenski SA, Peters KW, Alley DE, et al. The FNIH sarcopenia project: rationale, study description, conference recommendations, and final estimates. J Gerontol A Biol Sci Med Sci 2014;69:547-558.

15. Cawthon PM, Peters KW, Shardell MD, et al. Cutpoints for low appendicular lean mass that identify older adults with clinically significant weakness. J Gerontol A Biol Sci Med Sci 2014;69:567-575. 
16. Kim KM, Jang HC, Lim S. Differences among skeletal muscle mass indices derived from height-, weight-, and body mass index-adjusted models in assessing sarcopenia. Korean J Intern Med 2016;31:643-650.

17. Al Snih S, Markides KS, Ottenbacher KJ, Raji MA. Hand grip strength and incident ADL disability in elderly Mexican Americans over a seven-year period. Aging Clin Exp Res 2004;16:481-486.

18. Guralnik JM, Simonsick EM, Ferrucci L, et al. A short physical performance battery assessing lower extremity function: association with self-reported disability and prediction of mortality and nursing home admission. J Gerontol 1994;49:M85-M94.

19. Guralnik JM, Ferrucci L, Pieper CF, et al. Lower extremity function and subsequent disability: consistency across studies, predictive models, and value of gait speed alone compared with the short physical performance battery. J Gerontol A Biol Sci Med Sci 2000;55:M221-M231.

20. Peel NM, Kuys SS, Klein K. Gait speed as a measure in geriatric assessment in clinical settings: a systematic review. J Gerontol A Biol Sci Med Sci 2013;68:39-46.

21. Abbatecola AM, Paolisso G, Fattoretti P, et al. Discovering pathways of sarcopenia in older adults: a role for insulin resistance on mitochondria dysfunction. J Nutr Health Aging 2011;15:890-895.

22. Bijlsma AY, Meskers CG, van Heemst D, Westendorp RG, de Craen AJ, Maier AB. Diagnostic criteria for sarcopenia relate differently to insulin resistance. Age (Dordr) 2013;35:2367-2375.

23. Choi KM. Sarcopenia and sarcopenic obesity. Endocrinol Metab (Seoul) 2013;28:86-89.

24. Wang C, Bai L. Sarcopenia in the elderly: basic and clinical issues. Geriatr Gerontol Int 2012;12:388-396.

25. Jensen GL. Inflammation: roles in aging and sarcopenia. JPEN J Parenter Enteral Nutr 2008;32:656-659.

26. Meng SJ, Yu LJ. Oxidative stress, molecular inflammation and sarcopenia. Int J Mol Sci 2010;11:1509-1526.

27. Lang CH, Frost RA, Nairn AC, MacLean DA, Vary TC. TNF-alpha impairs heart and skeletal muscle protein synthesis by altering translation initiation. Am J Physiol Endocrinol Metab 2002;282:E336-347.

28. Schaap LA, Pluijm SM, Deeg DJ, Visser M. Inflammatory markers and loss of muscle mass (sarcopenia) and strength. Am J Med 2006;119:526.e9-526.e17.

29. McPherron AC, Lawler AM, Lee SJ. Regulation of skeletal muscle mass in mice by a new TGF-beta superfamily member. Nature 1997;387:83-90.

30. Grobet L, Martin LJ, Poncelet D, et al. A deletion in the bovine myostatin gene causes the double-muscled phenotype in cattle. Nat Genet 1997;17:71-74.

31. White TA, LeBrasseur NK. Myostatin and sarcopenia: opportunities and challenges: a mini-review. Gerontology 2014;60:289-293.

32. Srikanthan P, Hevener AL, Karlamangla AS. Sarcopenia exacerbates obesity-associated insulin resistance and dysglycemia: findings from the National Health and Nutrition Examination Survey III. PLoS One 2010;5:e10805.

33. Kim TN, Park MS, Yang SJ, et al. Prevalence and determinant factors of sarcopenia in patients with type 2 diabetes: the Korean Sarcopenic Obesity Study (KSOS). Diabetes Care 2010;33:1497-1499.

34. Jang HC. Sarcopenia, frailty, and diabetes in older adults. Diabetes Metab J 2016;40:182-189.

35. Hong HC, Hwang SY, Choi HY, et al. Relationship between sarcopenia and nonalcoholic fatty liver disease: the Korean Sarcopenic Obesity Study. Hepatology 2014;59:1772-1778.

36. Han K, Park YM, Kwon HS, et al. Sarcopenia as a determinant of blood pressure in older Koreans: findings from the Korea National Health and Nutrition Examination Surveys (KNHANES) 2008-2010. PLoS One 2014;9:e86902.

37. Snijder MB, Henry RM, Visser M, et al. Regional body composition as a determinant of arterial stiffness in the elderly: The Hoorn Study. J Hypertens 2004;22:2339-2347.

38. Sanada K, Iemitsu M, Murakami H, et al. Adverse effects of coexistence of sarcopenia and metabolic syndrome in Japanese women. Eur J Clin Nutr 2012;66:1093-1098.

39. Wijnhoven HA, Snijder MB, van Bokhorst-de van der Schueren MA, Deeg DJ, Visser M. Region-specific fat mass and muscle mass and mortality in community-dwelling older men and women. Gerontology 2012;58:32-40.

40. Han SS, Kim KW, Kim KI, et al. Lean mass index: a better predictor of mortality than body mass index in elderly Asians. J Am Geriatr Soc 2010;58:312-317.

41. Wannamethee SG, Shaper AG, Lennon L, Whincup PH. Decreased muscle mass and increased central adiposity are independently related to mortality in older men. Am J Clin Nutr 2007;86:1339-1346.

42. Atkins JL, Whincup PH, Morris RW, Lennon LT, Papacosta O, Wannamethee SG. Sarcopenic obesity and risk of cardiovascular disease and mortality: a population-based cohort study of older men. J Am Geriatr Soc 2014;62:253260. 
43. Hirani V, Blyth F, Naganathan V, et al. Sarcopenia is associated with incident disability, institutionalization, and mortality in community-dwelling older men: the concord health and ageing in men project. J Am Med Dir Assoc 2015;16:607-613.

44. Kim JH, Lim S, Choi SH, et al. Sarcopenia: an independent predictor of mortality in community-dwelling older Korean men. J Gerontol A Biol Sci Med Sci 2014;69:12441252.

45. Filippin LI, Teixeira VN, da Silva MP, Miraglia F, da Silva FS. Sarcopenia: a predictor of mortality and the need for early diagnosis and intervention. Aging Clin Exp Res 2015;27:249-254.

46. Baumgartner RN. Body composition in healthy aging. Ann N Y Acad Sci 2000;904:437-448.

47. Lim KI, Yang SJ, Kim TN, et al. The association between the ratio of visceral fat to thigh muscle area and metabolic syndrome: the Korean Sarcopenic Obesity Study (KSOS). Clin Endocrinol (Oxf) 2010;73:588-594.

48. Kim TN, Park MS, Lim KI, et al. Relationships between sarcopenic obesity and insulin resistance, inflammation, and vitamin D status: the Korean Sarcopenic Obesity Study. Clin Endocrinol (Oxf) 2013;78:525-532.

49. Kim TN, Choi KM. The implications of sarcopenia and sarcopenic obesity on cardiometabolic disease. J Cell Biochem 2015;116:1171-1178.

50. Zamboni M, Mazzali G, Fantin F, Rossi A, Di Francesco V. Sarcopenic obesity: a new category of obesity in the elderly. Nutr Metab Cardiovasc Dis 2008;18:388-395.

51. Gregor MF, Hotamisligil GS. Inflammatory mechanisms in obesity. Annu Rev Immunol 2011;29:415-445.

52. Kim TN, Park MS, Ryu JY, et al. Impact of visceral fat on skeletal muscle mass and vice versa in a prospective cohort study: the Korean Sarcopenic Obesity Study (KSOS). PLoS One 2014;9:e115407.

53. Kohara K. Sarcopenic obesity in aging population: current status and future directions for research. Endocrine 2014;45:15-25.
54. Stenholm S, Harris TB, Rantanen T, Visser M, Kritchevsky SB, Ferrucci L. Sarcopenic obesity: definition, cause and consequences. Curr Opin Clin Nutr Metab Care 2008;11:693-700.

55. Lim S, Kim JH, Yoon JW, et al. Sarcopenic obesity: prevalence and association with metabolic syndrome in the Korean Longitudinal Study on Health and Aging (KLoSHA). Diabetes Care 2010;33:1652-1654.

56. Chung JY, Kang HT, Lee DC, Lee HR, Lee YJ. Body composition and its association with cardiometabolic risk factors in the elderly: a focus on sarcopenic obesity. Arch Gerontol Geriatr 2013;56:270-278.

57. Kim TN, Park MS, Kim YJ, et al. Association of low muscle mass and combined low muscle mass and visceral obesity with low cardiorespiratory fitness. PLoS One 2014;9:e100118.

58. Lu CW, Yang KC, Chang HH, Lee LT, Chen CY, Huang KC. Sarcopenic obesity is closely associated with metabolic syndrome. Obes Res Clin Pract 2013;7:e301-e307.

59. Messier V, Karelis AD, Lavoie ME, et al. Metabolic profile and quality of life in class I sarcopenic overweight and obese postmenopausal women: a MONET study. Appl Physiol Nutr Metab 2009;34:18-24.

6o. dos Santos EP, Gadelha AB, Safons MP, Nobrega OT, Oliveira RJ, Lima RM. Sarcopenia and sarcopenic obesity classifications and cardiometabolic risks in older women. Arch Gerontol Geriatr 2014;59:56-61.

61. Stephen WC, Janssen I. Sarcopenic-obesity and cardiovascular disease risk in the elderly. J Nutr Health Aging 2009;13:460-466.

62. Batsis JA, Mackenzie TA, Barre LK, Lopez-Jimenez F, Bartels SJ. Sarcopenia, sarcopenic obesity and mortality in older adults: results from the National Health and Nutrition Examination Survey III. Eur J Clin Nutr 2014;68:10011007.

63. Tian S, Xu Y. Association of sarcopenic obesity with the risk of all-cause mortality: a meta-analysis of prospective cohort studies. Geriatr Gerontol Int 2016;16:155-166. 